\title{
News from the CLINAM Foundation
}

DOI 10.1515/ejnm-2014-0039

Dear Readers

Next June the European Foundation for Clinical Nanomedicine will hold its eighth European Summit for Clinical Nanomedicine and Targeted Medicine. With more than 500 participants from 42 countries, the last Summit was an overwhelming event.

Since last year the Summit has become a worldwide interdisciplinary neutral Nanomedicine platform. Our major goal is to support development and application of Nanomedicine and Targeted Medicine by elucidating the field in science, clinics, engineering and the ways to translation and industrial production. The CLINAM Summit has achieved a unique position in bringing together all stakeholders relevant to the field of Nanomedicine and Targeted Medicine.

Year after year, CLINAM rendered innovative novel topics but during the past 2 years the advances in development were so extensive and we see now a remarkable acceleration of development and new acceptance of Nanomedicine. During the last CLINAM Summit, for the first time, the regulatory authorities from all continents met in Basel. Under the lead of the European Medicines Agency (EMA) based in London, the Food and Drug Administration (FDA) from the United States and the responsible agency from Japan, a closed workshop for regulatory authorities was organized. The workshop was opened at the end for all participants of CLINAM. During that open discussion there was no doubt that a harmonization process started that will bring all stakeholders of the highly interdisciplinary Nanomedicine into a trustful cooperation, leading in the near future to standards and definitions and a better and faster translation process. It was also very remarkable, that the large pharmaceutical companies showed a substantial engagement in the field this time and more than 50 participants came from industry. The next summit looks promising and many novel aspects of the developing field will be debated and explained. This time the subheading of the Summit will be "The translation to Knowledge Based Nanomedicine”. There is now a huge need to identify the suitable pathways that come from the initial discovery to the product development to the benefit of the patient. The pathway from the innovative idea to the application, the development, regulatory approval and commercialization of a nanomedical drug is complex and first requires understanding the underlying cause of the disease. The translation process from research findings to applications in Nanomedicine and Targeted Medicine is developing and is currently on the agenda of all stakeholders. The decision makers in pharmaceutical companies have started to appreciate the quality and potential of Nanomedicine and Targeted Medicine for significantly increasing the output of diagnostics and therapeutic products with reduced side effects.

In the next decades, medicine will experience a transformation to personalized diagnosis and treatment, taking the individual aspects of the patient and his/her disease into consideration. High-resolution molecular profiling techniques have played a key role, these have been developed and will become inexpensive through Nanomedicine. The CLINAM Summit offers excellent opportunities for the huge number of organizations working towards this goal worldwide, to exchange expertise and to launch joint ventures for further acceleration of the processess. As during the last years the scientific committee of the CLINAM voted for three predominant fields in medicine to be focused on. The 2015's Summit concentrates on: Infection and Inflammation, Cancer, and Diabetes. At CLINAM 8/2015 you will have the unique opportunity to present and discuss your work with experts from all fields within the realm of Nanomedicine and Targeted Medicine, including regulatory authorities, industrial, academic, and clinical experts. The most comprehensive feedback that you can obtain will be by presenting small talks on your work. The Call for papers will be online as from December and the first preliminary programme will be online in February at www.clinam.org.

I take this opportunity to wish you all a very successful 2015 and hope to see many of you in Basel from June 28th until July 1st at the Swiss Trade Fair Congress Center. See you next year!

\section{Dr. Beat Löffler, MA}

CEO of the European Foundation for Clinical Nanomedicine 